\title{
Five-year follow-up of a woman with pregnancy and lactation-associated osteoporosis and vertebral fractures
}

This article was published in the following Dove Press journal:

Therapeutics and Clinical Risk Management

6 April 2012

Number of times this article has been viewed

\author{
Jun Iwamoto' \\ Yoshihiro Sato ${ }^{2}$ \\ Mitsuyoshi Uzawa ${ }^{3}$ \\ Hideo Matsumoto' \\ 'Institute for Integrated Sports \\ Medicine, Keio University School \\ of Medicine, ${ }^{2}$ Department \\ of Neurology, Mitate Hospital, \\ Fukuoka, ${ }^{3}$ Department of Orthopaedic \\ Surgery, Keiyu Orthopaedic Hospital, \\ Gunma, Japan
}

\begin{abstract}
We report the 5-year follow-up of a young woman who developed vertebral fractures after pregnancy and lactation and was treated with active vitamin D hormone. A 32-year-old Japanese woman consulted us because of acute lower back pain caused by L2 and L5 vertebral fractures after pregnancy and lactation. Following cessation of breast-feeding, analgesia, bed rest, and wearing of a hard brace, her lower back pain disappeared within 2 months. After 5 years of treatment with alfacalcidol $1 \mu \mathrm{g}$ daily, the lumbar spine (L1, L3, L4) bone mineral density increased by $21.4 \%$ following vigorous reductions in bone turnover markers. No osteoporotic fractures occurred, and the vertebral fractures healed. The patient experienced no side effects, including hypercalcemia. Thus, the present case report shows long-term changes in bone turnover markers and lumbar spine bone mineral density, as well as long-term safety of alfacalcidol treatment in a young woman with pregnancy and lactation-associated osteoporosis and vertebral fractures.
\end{abstract}

Keywords: young woman, pregnancy, alfacalcidol, bone mineral density, osteoporosis, vertebral fracture

\section{Background}

Pregnancy and lactation-associated osteoporosis are rare conditions characterized by significant changes in calcium and bone homeostasis arising from the increased fetal demand for calcium for skeletal bone calcification and the subsequent occurrence of fragility fractures during late pregnancy or during the postpartum period. ${ }^{1,2}$ To date, no good evidence to guide medicinal interventions is available in patients with pregnancy and lactation-associated osteoporosis.

Given that calcium imbalances during pregnancy and the lactation period are not surprising, ${ }^{1,2}$ therapeutic options primarily include calcium and vitamin D supplementation in an appropriate clinical setting. ${ }^{3}$ In Japan, nutritional calcium and vitamin D supplements are not widely available, and active vitamin D hormones are alternatively used. We report the 5-year follow-up of a young woman with acute lower back pain caused by lumbar vertebral fractures after pregnancy and lactation who was treated with active vitamin D hormone.

\section{Case report}

A young 32-year-old Japanese woman consulted our clinic because of nontraumatic acute lower back pain. Her height was $155 \mathrm{~cm}$, her body weight was $57 \mathrm{~kg}$, and her body mass index was $23.7 \mathrm{~kg} / \mathrm{m}^{2}$. She had given birth to a baby 3 months before her visit to our clinic and had breastfed the baby for 3 months. She had no past history 


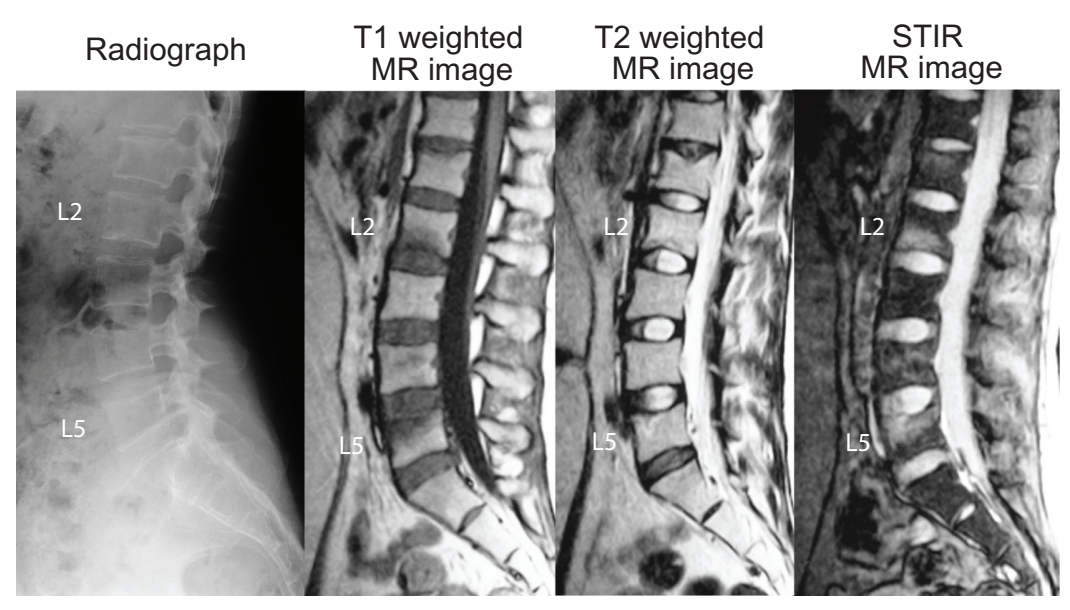

Figure I Radiographs and MR images of the lumbar spine - lateral views before treatment.

Notes: Radiographs of the lumbar spine did not show any obvious vertebral fractures (morphometric fractures) in the lumbar spine. However, TI-weighted MR images identified low-intensity areas and T2-weighted and STIR MR images identified high-intensity areas at the L2 and L5 vertebral bodies.

Abbreviations: MR, magnetic resonance; STIR, short-tau inversion-recovery.

of metabolic bone disease and had never taken medicine affecting bone metabolism. She did not have any clinical risk factors for fractures, including current smoking, a maternal history of hip fractures, alcohol consumption $\geq 2$ units daily, age $\geq 75$ years, leanness (body mass index $\leq 18.5 \mathrm{~kg} / \mathrm{m}^{2}$ ), or a history of steroid use. ${ }^{4}$

Although radiographs of the lumbar spine did not show any obvious vertebral (morphometric) fractures in the lumbar spine, ${ }^{5,6}$ magnetic resonance imaging detected clinical vertebral fractures at the L2 and L5 spine (Figure 1). She was hospitalized for treatment. She stopped lactation and was restricted to bed rest with the occasional use of nonsteroidal anti-inflammatory drugs. After 2 weeks of bed rest, her lower back pain had significantly improved, the occasional use of non-steroidal anti-inflammatory drugs was stopped, and rehabilitation was started, with the patient wearing a hard brace.

The lumbar spine bone mineral density (BMD) in the anteroposterior view was measured using dual-energy $\mathrm{x}$-ray absorptiometry, and biochemical analyses were performed. Table 1 shows the baseline bone mineral density and biochemical markers. The patient was diagnosed as having high bone turnover osteoporosis. ${ }^{5,6}$ Treatment with alfacalcidol $1 \mu \mathrm{g}$ daily was started, and the patient was discharged from hospital.

\section{Outcome of 5 years of treatment with alfacalcidol}

The patient's lower back pain was alleviated after 2 months of treatment, bony union was confirmed, and the hard brace was removed. The lumbar spine BMD increased from the baseline value (Figure 2) and reached $0.906 \mathrm{~g} / \mathrm{cm}^{2}$, corresponding to $92 \%$ of the age-matched control. Figures 3 and 4 show changes in biochemical markers. Serum alkaline phosphatase, bone alkaline phosphatase, parathyroid levels, and urinary $\mathrm{N}$-terminal telopeptide levels were within the normal range after 5 years of alfacalcidol treatment.

At 5 years, radiographs of the thoracic and lumbar spine revealed no evidence of new thoracic or lumbar morphometric vertebral fractures, ${ }^{5,6}$ and the L2 and L5 vertebral fractures had healed with small height loss of the vertebral bodies (Figure 5). During the 5-year period of alfacalcidol treatment, non-vertebral osteoporotic fractures did not occur. Furthermore, neither hypercalcemia nor adverse effects were observed. The treatment was discontinued when the patient became pregnant again after 5 years of treatment with alfacalcidol.

Table I BMD and biochemical markers before treatment

\begin{tabular}{lll}
\hline & & Normal range \\
\hline Lumbar spine BMD $\left(\mathrm{g} / \mathrm{cm}^{2}\right)$ & 0.746 & \\
\% YAM in BMD & 74 & \\
\% AMC in BMD & 75 & \\
Serum & & \\
$\quad$ Calcium $(\mathrm{mg} / \mathrm{dL})$ & 8.9 & $8.4-10.2$ \\
Phosphorus $(\mathrm{mg} / \mathrm{dL})$ & 4.1 & $2.5-4.5$ \\
ALP $(\mathrm{IU} / \mathrm{L})$ & 398 & $100-340$ \\
Intact PTH $(\mathrm{pg} / \mathrm{mL})$ & 10.3 & $10-66$ \\
BAP $(\mu \mathrm{g} / \mathrm{L})$ & 49.7 & $3.8-22.6$ \\
Urine & & \\
NTX $(\mathrm{nM} B C E / \mathrm{mM} \mathrm{Cr})$ & 85.3 & $9.3-54.3$ \\
Calcium/Cr & 0.098 & $<0.3$ \\
\hline
\end{tabular}

Abbreviations: BMD, bone mineral density; YAM, young adult mean; AMC, agematched control; ALP, alkaline phosphatase; PTH, parathyroid hormone; BAP, bonespecific alkaline phosphatase; NTX, cross linked N-terminal telopeptides of type I collagen; BCE, bone collagen equivalent; $\mathrm{Cr}$, creatinine. 


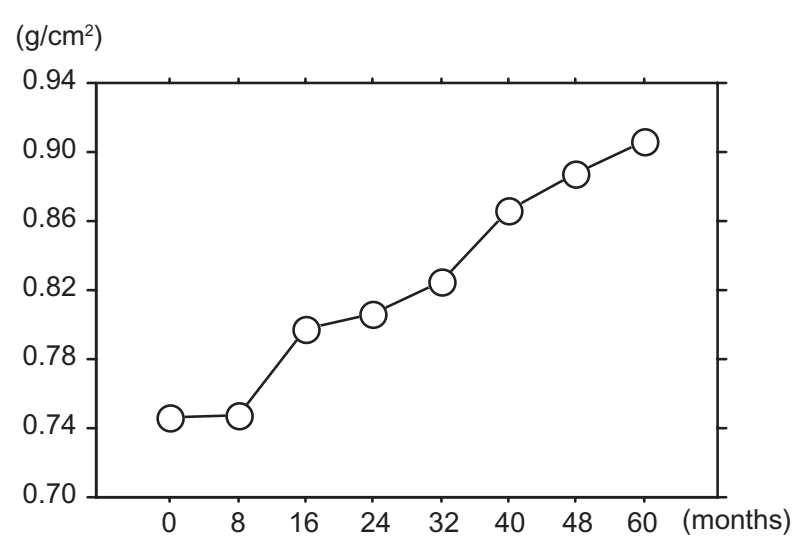

Figure 2 Changes in lumbar spine BMD.

Notes: The lumbar spine BMD increased from the baseline value. The increase in the lumbar spine BMD after 5 years of treatment with alfacalcidol was $21.4 \%$.

Abbreviation: BMD, bone mineral density.

\section{Discussion}

Pregnancy and lactation place a large amount of stress on maternal calcium homeostasis. Physiologically, there are increased calcium requirements during pregnancy, particularly during the third trimester when rapid mineralization of the fetal skeleton occurs. ${ }^{7}$ This increase is compensated for
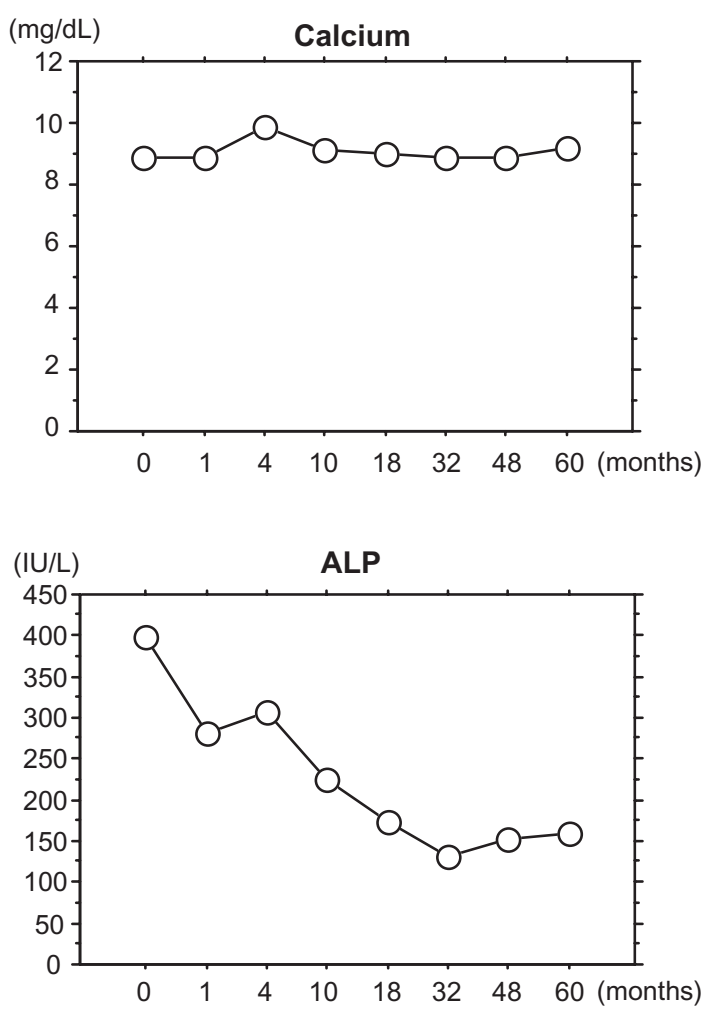

by increased intestinal absorption, reduced urinary calcium excretion, and increased bone resorption. ${ }^{8}$ The degree of the reduction in BMD during pregnancy, in the range of $2 \%-4 \%$, depending on the site of measurement. ${ }^{9}$ During lactation, the reduction in BMD approximates the duration of lactation, with significant reductions in both the hip and lumbar spine. ${ }^{7}$ Approximately 5\% of the BMD is lost in the lumbar spine and femoral neck during lactation lasting for 6 months or longer. ${ }^{10}$ In the current patient with low BMD, bone turnover increased, but release of excess calcium from this resorptive process in terms of hypercalcemia and hypercalciuria was not observed. Parathyroid gland function was mildly suppressed, probably because of physiological changes in intestinal calcium absorption and renal calcium reabsorption.

Patients with pregnancy and lactation-associated osteoporosis can expect to return to normal 6-12 months after breast-feeding has stopped. ${ }^{7}$ Although the bone turnover markers were normalized, the lumbar spine BMD remained low. This finding suggests a pre-existing low lumbar spine BMD.

A couple of studies have shown that the effects of one year of treatment with alfacalcidol on the lumbar spine BMD and
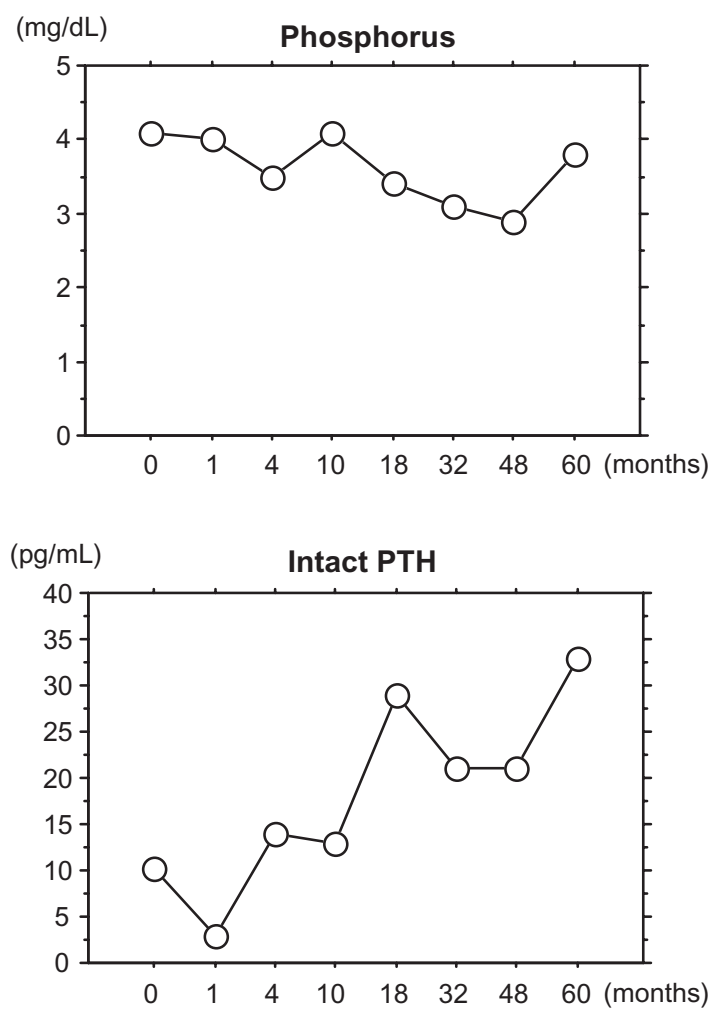

Figure 3 Changes in serum calcium, phosphorus, ALP, and intact PTH.

Notes: The serum ALP levels tended to decrease, and the serum intact PTH levels tended to increase from the baseline values. The serum levels of calcium and phosphorus stayed within the normal ranges. The decrease in the serum ALP levels after 5 years of treatment with alfacalcidol was $59.8 \%$. The increase in the serum intact PTH level was $220 \%$.

Abbreviations: ALP, alkaline phosphatase; PTH, parathyroid hormone. 


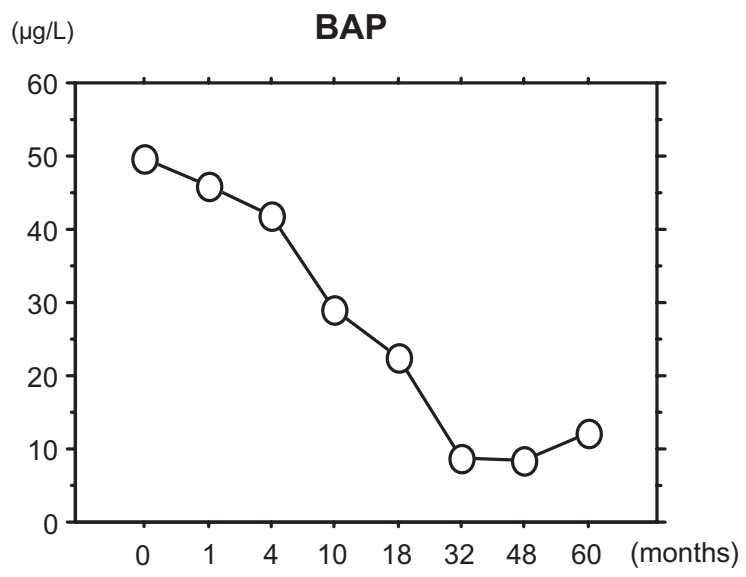

(nM BCE/mM Cr) Urinary NTX

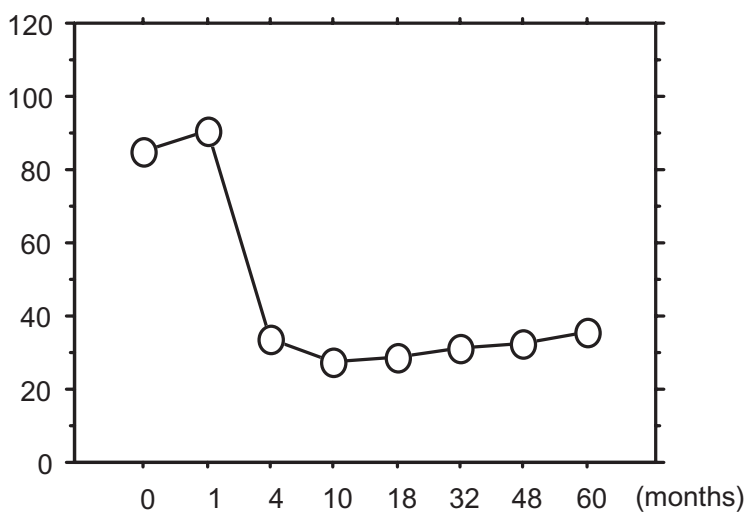

Figure 4 Changes in bone turnover markers.

Notes: The serum BAP levels and urinary NTX levels tended to decrease from the baseline values. The decrease in the serum BAP levels and the urinary NTX levels after 5 years of treatment with alfacalcidol were $75.7 \%$ and $58.3 \%$, respectively.

Abbreviations: BAP, bone-specific alkaline phosphatase; NTX, cross-linked N-terminal telopeptides of type I collagen.

bone turnover markers were modest among Japanese patients with involutional and postmenopausal osteoporosis. ${ }^{11,12}$ Thus, the dramatic changes in bone turnover markers and intact parathyroid hormone in the current patient could be mostly attributed to the natural course of pregnancy and lactationassociated osteoporosis, combined with the cessation of breast-feeding. However, there may have been small effects of alfacalcidol on bone turnover markers and lumbar spine BMD. No osteoporotic fractures occurred, and neither hypercalcemia nor adverse effects were observed. Thus, the present case report showed long-term changes in bone turnover markers and lumbar spine BMD, as well as the long-term safety of alfacalcidol treatment in a young woman with pregnancy and lactation-associated osteoporosis and vertebral fractures.

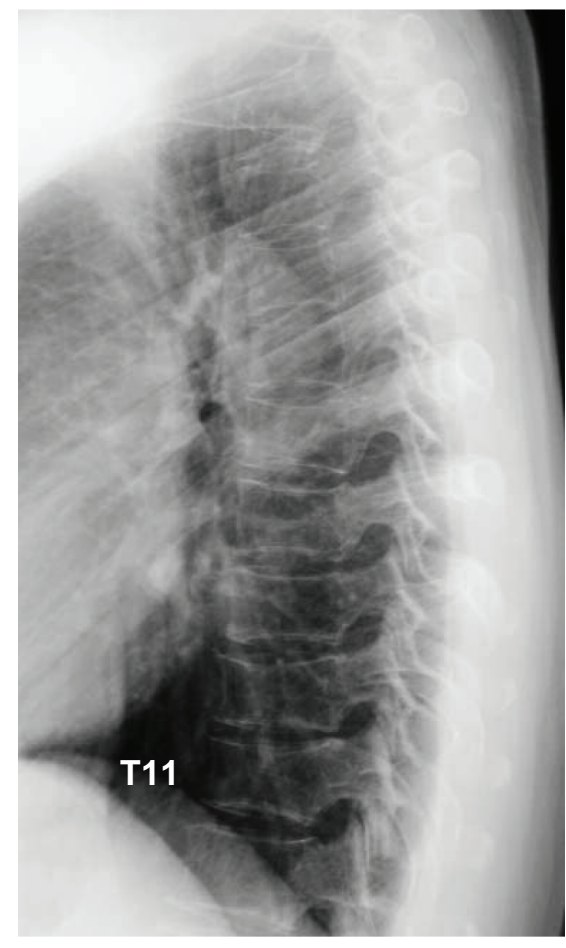

Thoracic spine

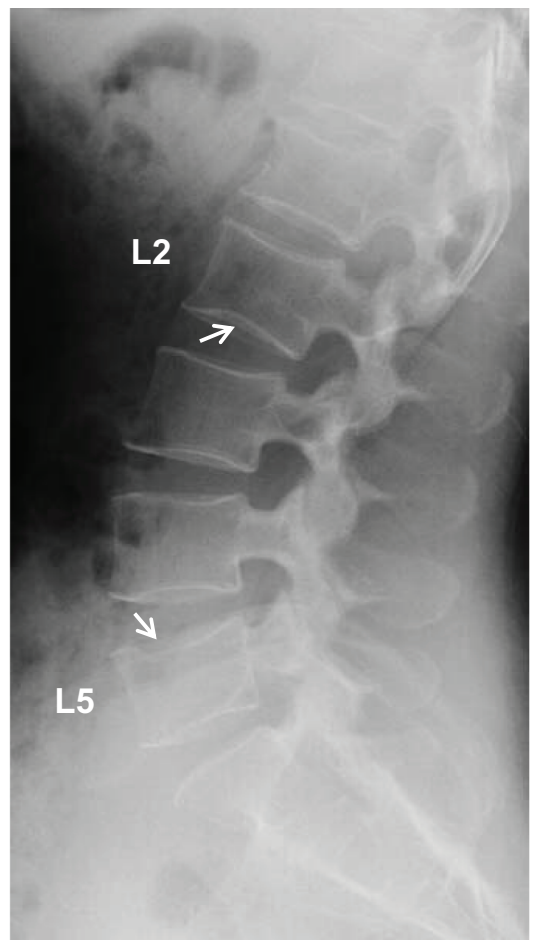

\section{Lumbar spine}

Figure 5 Radiographs of the thoracic and lumbar spine - lateral views after 5 years of treatment.

Notes: After 5 years of alfacalcidol treatment, radiographs of the thoracic and lumbar spine revealed no evidence of new thoracic or lumbar vertebral fractures, and the $\mathrm{L} 2$ and L5 vertebral fractures had healed. 
In conclusion, the present case report shows long-term changes in bone turnover markers and lumbar spine BMD, as well as long-term safety of alfacalcidol treatment, in a young woman with pregnancy and lactation-associated osteoporosis and vertebral fractures.

\section{Disclosure}

The authors report no conflict of interest in this work.

\section{References}

1. Rizzoli R, Bonjour JP. Pregnancy-associated osteoporosis. Lancet. 1996;347:1274-1276.

2. O'Sullivan SM, Grey AB, Singh R, et al. Bisphosphonates in pregnancy and lactation-associated osteoporosis. Osteoporos Int. 2006;17:1008-1012.

3. Michalakis K, Peitsidis P, Ilias I. Pregnancy- and lactation-associated osteoporosis: a narrative mini-review. Endocr Regul. 2011;45:43-47.

4. Orimo H. Japanese guideline for prevention and treatment of osteoporosis. Risk factors for fragility fractures and their assessment. Life Science. 2011:38-39. Japanese.
5. Orimo H, Sugioka Y, Fukunaga M, et al. Diagnostic criteria of primary osteoporosis. J Bone Miner Metab. 1998;16:139-150.

6. Orimo H, Hayashi Y, Fukunaga M, et al. Diagnostic criteria for primary osteoporosis: year 2000 revision. J Bone Miner Metab. 2001;19:331-337.

7. Tran HA, Petrovsky N. Pregnancy-associated osteoporosis with hypercalcaemia. Intern Med J. 2002;32:481-485.

8. Yoon BK, Lee JW, Choi DS, et al. Changes in biochemical bone markers during pregnancy and puerperium. J Korean Med Sci. 2000;15:189-193.

9. Khovidhunkit W, Epstein S. Osteoporosis in pregnancy. Osteoporos Int. 1996;6:345-354.

10. Sowers MF, Hollis BW, Shapiro B, et al. Elevated parathyroid hormonerelated peptide associated with lactation and bone density loss. JAMA. 1996;276:549-554.

11. Orimo H, Shiraki M, Hayashi Y, et al. Effects of $1 \alpha$-hydroxyvitamin $\mathrm{D}_{3}$ on lumbar bone mineral density and vertebral fractures in patients with osteoporosis. Calcif Tissue Int. 1994;54:370-376.

12. Shiraki M, Kushida K, Fukunaga M, et al. A double-masked multicenter comparative study between alendronate and alfacalcidol in Japanese patients with osteoporosis. Osteoporos Int. 1999;10:183-192.
Therapeutics and Clinical Risk Management

\section{Publish your work in this journal}

Therapeutics and Clinical Risk Management is an international, peerreviewed journal of clinical therapeutics and risk management, focusing on concise rapid reporting of clinical studies in all therapeutic areas, outcomes, safety, and programs for the effective, safe, and sustained use of medicines. This journal is indexed on PubMed Central, CAS,

\section{Dovepress}

EMBase, Scopus and the Elsevier Bibliographic databases. The manuscript management system is completely online and includes a very quick and fair peer-review system, which is all easy to use. Visit http://www.dovepress.com/testimonials.php to read real quotes from published authors.

Submit your manuscript here: http://www.dovepress.com/therapeutics-and-clinical-risk-management-journal 